\title{
Efeito da via de parto sobre a força muscular do assoalho pélvico
}

\author{
The influence of the delivery route on pelvic floor muscle strength
}

\author{
Angélica Mércia Pascon Barbosa ${ }^{1}$, Lidia Raquel de Carvalho ${ }^{2}$, Anice Maria Vieira de Camargo Martins ${ }^{3}$, \\ Iracema de Mattos Paranhos Calderon ${ }^{4}$, Marilza Vieira Cunha Rudge ${ }^{5}$
}

\section{RESUM0}

Objetivo: analisar a influência da via de parto sobre a força muscular do assoalho pélvico (FM-AP). Métodos: estudo clínico de corte transversal, para avaliar a FM-AP pelo teste da avaliação da força do assoalho pélvico (AFA) e uso do perineômetro em primíparas, entre 20-30 anos de idade, 4-6 meses pós-parto. A contração, medida pelos dois testes, foi classificada em: zero - ausência, um - leve, dois - moderada e três - normal, sustentada por 6 segundos. Avaliaram-se 94 mulheres, entre 20 e 30 anos, divididas em três grupos: pós-parto vaginal $(n=32)$; pós-cesárea $(n=32)$ e nulíparas $(n=30)$. A variável independente foi a via de parto e a dependente, a FM-AP. A comparação entre os graus de contração foi realizada pelo teste de KruskalWallis e o teste de Dunn para comparações múltiplas; a influência da via de parto pelo teste $\chi^{2}$, o risco relativo (RR) para alteração da FM-AP e o coeficiente kappa para avaliar equivalência entre os testes. Resultados: a mediana e $1^{\circ}$ e $3^{\circ}$ quartil da FM-AP foram menores ( $\mathrm{p}=0,01)$ pós-parto vaginal $(2,0 ; 1-2)$ e intermediários pós-cesárea $(2,0 ; 2-3)$ em relação às nulíparas $(3,0 ; 2-3)$, tanto analisadas pelo AFA como pelo perineômetro. Aumentou o RR de exame alterado pós-parto vaginal (RR=2,5; IC 95\%: 1,3-5,0; $p=0,002) ;(R R=2,3 ; I C ~ 95 \%: 1,2-4,3 ; p=0,005)$ e pós-cesárea $(R R=1,5 ; I C ~ 95 \%: 0,94-2,57 ; p=0,12) ;(R R=1,3 ; I C$ 95\%: 0,85-2,23; $\mathrm{p}=0,29)$ pelo PFSE e perineômetro, respectivamente. Conclusões: o parto vaginal diminuiu a força muscular do AP de primíparas quando comparado com os casos submetidos à cesárea e com as nulíparas.

PALAVRAS-CHAVE: Assoalho pélvico; Parto normal; Cesárea

\section{ABSTRACT}

Purpose: to evaluate the influence of the delivery route on pelvic floor (PF) muscle strength. Methods: a cross-sectional study was conducted to evaluate PF muscle strength by the pelvic floor strength evaluation (PFSE) test and perineometer in primiparous patients aged 20 to 30 years 4 to 6 months after delivery. The categorization was: zero - lack of muscle contraction; one - weak contraction; two - moderate contraction not sustained for $6 \mathrm{~s}$ and three - normal contraction sustained for $6 \mathrm{~s}$. A total of 94 patients were divided into there groups based on prior delivery route. They were: 32 patients with vaginal delivery with singleton cephalic presentation; 32 patients with cesarean delivery, and 30 nulliparous patients as a control group. The independent variable was delivery route and the dependent one was the muscle strength of the PF. Comparison between contraction levels was performed by Kruskal-Wallis and Dunn multiple comparison tests and the influence of delivery method was tested by $\chi^{2}$. Confidence interval of $95 \%$ was obtained for relative risk (RR) of PF muscle strength changes and kappa statistics. Results: the 1st and 3rd quartiles of delivery route regarding PF muscle strength were lower $(\mathrm{p}=0.01)$ for vaginal delivery $(2.0 ; 1-2)$ and intermediate for cesarean section $(2.0 ; 2-3)$ compared to the nulliparous $(3.0 ; 2-3)$ by the PFSE test and perineometer. RR of the altered examination was increased after vaginal delivery $(\mathrm{RR}=2.58 ; \mathrm{CI}$ 95\%: 1.32-5.04, $\mathrm{p}=0.002) ;(\mathrm{RR}=2.31$; CI 95\%: 1.24-4.32, $\mathrm{p}=0.005)$, and after cesarean section $(\mathrm{RR}=1.56 ; \mathrm{CI} 95 \%: 0.94-2.57, \mathrm{p}=$ 0.12); $(\mathrm{RR}=1.38$; CI 95\%: 0.85-2.23, $\mathrm{p}=0.29)$ by AFA and perineometer, respectively. Conclusions: vaginal delivery decreased PF muscle strength when compared with cesarean delivery and control groups.

KEYWORDS: Pelvic floor; Natural childbirth; Cesarean section

Universidade Estadual Paulista "Julio de Mesquita Filho" - UNESP - Botucatu (SP) - Brasil.

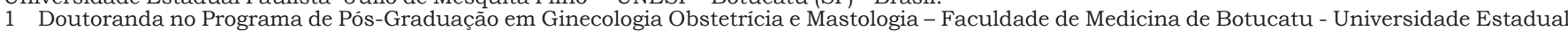
Paulista "Júlio de Mesquita Filho" - UNESP - Botucatu (SP) - Brasil.

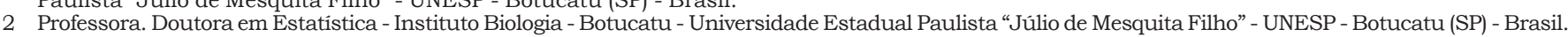

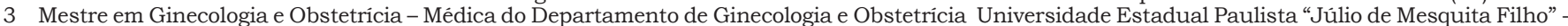
UNESP - Botucatu (SP) - Brasil.

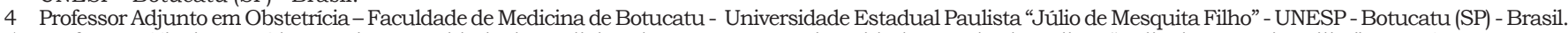

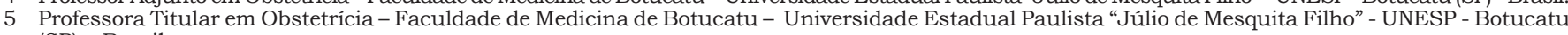
(SP) - Brasil.

Correspondência: Angélica Mércia Pascon Barbosa

Amador Bueno 630 - Vila Central - 19806-190 - Assis - SP - Telefone: (18) 3322-4433 - Fax: (18) 3324-9088 - e-mail: pasconbarbosa@uol.com.br 


\section{Introdução}

A gravidez e a via de parto são fatores de risco para alteração da força muscular do assoalho pélvico $(\mathrm{AP})^{1}$. O incremento do peso corporal materno e o peso do útero gravídico aumentam a pressão sobre a musculatura do AP na gestação ${ }^{1}$. O aumento do índice de massa corpórea (IMC) na gravidez, a multiparidade, o parto vaginal, o tempo prolongado do segundo período do parto e a episiotomia são fatores que diminuem a força dos músculos do $\mathrm{AP}^{2}$.

Na gestação, no trabalho de parto e no parto ocorrem mudanças na posição anatômica da pelve, na forma da musculatura pélvica, nas vísceras e no períneo. Estas alterações e a relação com a gravidez e parto podem ser explicadas pela deficiência do AP na gestação e no parto, pois a sobrecarga do períneo causa neuropatia do pudendo ${ }^{3}$. Os processos fisiológicos seqüenciais durante a gestação e o parto lesam o suporte pélvico, o corpo perineal e o esfincter anal e são fatores determinantes, a longo prazo, para o surgimento das perdas urinárias ${ }^{4}$.

As estruturas do AP feminino funcionam como uma unidade, sendo importante a relação anatômico-funcional entre todas as estruturas pélvicas para a manutenção da função normal. No trabalho de parto e parto, o músculo elevador do ânus pode ser lesado por dois mecanismos: por injúria direta, causada pela lesão mecânica ou decorrente da distensão do próprio músculo, e por injúria indireta, causada pela lesão do nervo que o supre. Quando o músculo é estirado em mais da metade de seu comprimento total, pode sofrer lesão grave. Durante o parto, as fibras do músculo elevador do ânus podem se alongar cerca de metade do seu comprimento para circundar a cabeça fetal ${ }^{5}$.

A lesão dos músculos pélvicos, dos nervos ou da fáscia parece ser conseqüência inevitável do parto vaginal, independente das distocias no parto. A vagina sofre menos dilatação e distensão que o colo do útero, mas é questionável se a lesão da fáscia endopélvica é mais grave que a do colo do útero. É pouco provável que os ligamentos cardinais e uterossacros sejam lesados de forma irreparável e é mais aceitável que a resposta à tração, notada após o parto, reflita a embebição gravídica dos tecidos e não sua lesão ou destruição ${ }^{5}$.

As lesões neurológicas dos músculos pélvicos são bem documentadas ${ }^{6}$. À medida que pequenos nervos são separados das fibras musculares, a habilidade contrátil dessas fibras musculares é diminuída e a função normal é perdida. O trauma pélvico materno relacionado ao parto descomplicado e considerado normal inclui tanto lesão nervosa reversível quanto irreversivel. A abertura do AP, para a passagem da cabeça fetal, é pequena e, por essa razão, a cabeça empurra o
AP para baixo até que tenha dilatado o suficiente para passar por ela ${ }^{5}$. Os relatos documentam essas alterações do AP após o parto vaginal.

O Brasil é um país com altas taxas de cesáreas, e as mulheres e os médicos usam o comprometimento do AP pós-parto vaginal como forma de estímulo para a realização de cesárea eletiva. A literatura tem discutido o direito das mulheres à cesárea eletiva ${ }^{7}$, as suas vantagens em relação à cesárea de urgência durante o trabalho de parto e as diferenças de custo ${ }^{8}$. Estudar o que acontece com a força muscular do AP pós-parto cesáreo e pós-parto vaginal pode ser argumento a ser usado na discussão dos benefícios da via alta ou da via baixa.

A força muscular do AP pode ser mensurada e, conseqüentemente, medido o grau de força muscular ${ }^{9}$. Existem várias metodologias usadas, dentre elas: a eletroneuromiografia, o teste de condução motora, os cones vaginais, o myofeedback ou perineômetro e a AFA (avaliação da força do assoalho pélvico $)^{10-12}$. Os mais utilizados são o perineômetro e a AFA porque são de uso rotineiro na clínica, a instrumentação é simples, de baixo custo na execução, tem boa confiabilidade técnica e boa aceitabilidade pelas mulheres ${ }^{12-14}$.

Este trabalho teve como objetivo determinar se a via de parto altera a força muscular do AP de primiparas, entre 4 e 6 meses pós-parto vaginal e cesárea.

\section{Métodos}

Desenvolvemos estudo clínico de corte transversal, baseado em protocolo preestabelecido, do exame da força muscular do AP de 94 mulheres. Foi realizado na Secretaria da Saúde na cidade de Assis (SP), entre 2002 e 2003.

Assis é cidade com, aproximadamente, 100.000 habitantes, localizada na região da DIR VIII do estado de São Paulo, Brasil. Os partos foram no Hospital Regional de Assis, no Hospital e Maternidade de Assis, no Instituto de Atendimento à Mulher e na Santa Casa de Assis.

O cálculo do tamanho amostral foi baseado nos valores médios e de desvio-padrão, obtidos na literatura ${ }^{15}$, da variável idade materna, ou seja, valor médio de 28 anos de idade, desvio padrão igual a 10 e diferença esperada entre o grupo controle e os demais grupos em estudo de 7,2 anos. Foi utilizado o poder do teste de $80 \%$ e nível de significância de $5 \%$, e determinado o número mínimo de 30 mulheres por grupo.

Os critérios de inclusão foram: primíparas entre 20 e 30 anos de idade, gestação de termo, com parto vaginal com episiotomia ou cesárea, 4 a 6 meses pós-parto e nuliparas na mesma faixa etá- 
ria. Os critérios de exclusão foram mulheres com história de abortamento anterior, cirurgia abdominal prévia e ganho de mais de $22 \mathrm{~kg}$ na gestação.

Foram incluidas 64 mulheres que atenderam aos critérios de inclusão, sendo 32 pós-parto vaginal e 32 pós-cesárea, e 30 nulíparas que atenderam aos critérios de inclusão.

Os grupos experimentais foram: Grupo G-PV, constituído de 32 primíparas pós-parto vaginal, cefálico com episiotomia, Grupo G-PC, com 32 primíparas pós-parto cesáreo, e Grupo G-NP, com 30 nuliparas. A variável independente foi a via de parto, sendo consideradas as vias vaginal e abdominal. A variável dependente foi a força muscular do AP, classificada pelos testes da AFA e pelo perineômetro. As variáveis de controle foram: idade entre 20 e 30 anos, divididas em três faixas etárias: 20 e 22, entre 23 e 26 e acima de 27 anos, idade gestacional ao nascimento, peso do recémnascido e tempo pós-parto.

As mulheres foram informadas da pesquisa, assinaram o termo de consentimento livre e esclarecido (TCLE) e preencheram questionário inicial, informando dados gerais referentes à gestação e ao parto. A idade gestacional ao nascimento foi calculada em semanas, pela data da última menstruação ${ }^{16}$. O peso do recém-nascido (em gramas) foi referido pela paciente.

A força muscular do AP foi obtida com a mulher colocada em posição ginecológica, com as regiões abdominal, assoalho pélvico e membros inferiores desnudos. Esta posição é a mais indicada por fornecer avaliação mais precisa da contração isolada da musculatura perineal ${ }^{17}$. A mulher foi, previamente, conscientizada para fazer a força como se necessitasse "segurar" a urina contraindo apenas os músculos do AP. O examinador calçou luvas para realizar os exames. O teste da AFA foi realizado pelo toque vaginal bidigital do examinador, que solicitou verbalmente a contração voluntária perineal; foram solicitadas três contrações com intervalo de um minuto entre uma e outra. O examinador permaneceu com o toque bidigital apenas durante as contrações. Avaliou-se o grau da força muscular em cada contração, classificando-o de zero: ausente, um: contração leve, dois: contração moderada não sustentada por 6 segundos e três: contração normal sustentada por seis segundo ${ }^{18}$. O resultado de cada contração foi registrado e o resultado final foi obtido pela média entre os valores dos três registros. O teste foi considerado normal quando recebeu classificação três e alterado para zero, um e dois.

Após descanso de quinze minutos, foi realizado o exame com o perineômetro, que é um eletromiógrafo de pressão, que registra contrações musculares do AP e traduz sua intensidade por sinais visuais numa escala numérica. É constituído por um sensor vaginal inflável recoberto por látex, um monitor com visor de leitura eletrônico e um transdutor de pressão ${ }^{17}$. O visor de leitura eletrônico tem escala numérica em $\mathrm{mmHg}$ com intervalos de 1,6 $\mathrm{mmHg}$. O estudo piloto permitiu a classificação em zero - não há indicação numérica, um - contração leve, com indicação numérica nos intervalos de 1,6 a 16,0 mmHg, dois - contração moderada, não sustentada por seis segundos, nos intervalos de 17,6 a 32,0 mmHg, e três contração normal sustentada por seis segundos, no intervalo de 33,6 a 46,4 mmHg. O teste foi considerado normal quando recebeu classificação três e alterado para zero, um e dois.

Com a mulher na posição ginecológica, a haste de látex, previamente revestida com preservativo descartável, foi introduzida na vagina. O nivel da pressão na escala foi zerado e foi solicitado à mulher a contração perineal voluntária mantida pelo maior tempo possivel, numa seqüência de três contrações, com intervalos de um minuto entre elas. Foram registrados os valores das três contrações e estimados os valores de pico, a média e a duração da contração. Verificou-se a média entre os valores dos três registros que foi utilizada como resultado para classificação.

\section{Análise estatistica}

As variáveis de controle, idade gestacional do parto, peso do recém-nascido, ganho de peso na gestação, tempo pós-parto e as três faixas etárias da idade materna (20-22; 23-27 e acima de 27 anos) foram comparadas pelo teste de $t$. A comparação dos graus de força muscular entre os três grupos experimentais, nos dois testes de avaliação funcional do AP, foi realizada utilizando um teste de KruskalWallis e o teste de Dunn para as comparações múltiplas. O nivel de significância utilizado foi de $95 \%$ de probabilidade. Os resultados das alterações da força muscular do AP foram analisados pelo teste do $\chi^{2}$ para verificação da influência da via de parto. O nível de significância utilizada foi de $\mathrm{p} \leq 0,05$ de probabilidade. Foi calculado o risco relativo (RR) de alteração da força muscular e o intervalo de confiança (IC) a $p \leq 0,05$ de exame alterado da musculatura do $\mathrm{AP}^{19}$. O coeficiente kappa foi calculado para avaliar a equivalência dos testes AFA e perineômetro. O presente trabalho foi aprovado pelo Comitê de Ética em Pesquisa da Faculdade de Medicina de Marília - FAMEMA em 25 de julho de 2002 e tomado ciência pelo Comitê de Ética em Pesquisa da Faculdade de Medicina de Botucatu-UNESP.

\section{Resultados}

A idade gestacional no momento do parto foi semelhante nos grupos G-PV $(30,9 \pm 1,1)$ e G-PC 
$(39,5 \pm 1,1)$; o ganho de peso na gestação foi semelhante $(13,8 \mathrm{~kg})$ entre os dois grupos. O peso dos recém-nascidos não mostrou diferença entre os dois grupos (G-PV=3070 \pm 346 e G-PC=3157 \pm 523 ). As mulheres foram avaliadas após 5,1 meses no grupo G-PV e após 4,9 meses no G-PC. Nas três faixas etárias consideradas (20-22; 23-27 e acima de 27) foi semelhante o percentual de nuliparas e primíparas pós-parto vaginal e pós-cesárea $(p=0,06)$. Esses resultados evidenciam a homogeneidade entre os grupos.

O parto vaginal e a cesárea diminuíram a força muscular do AP avaliada pelos testes AFA e perineômetro. A mediana e o $1^{\circ}$ e $3^{\circ}$ quartil da força muscular do AP foram menores no grupo pósparto vaginal $(2,0 ; 1-2)$ e intermediários $(2,02-3)$ no grupo pós-parto cesárea, tanto pela AFA como com o perineômetro $(p<0,05)$ em relação às nuliparas (2,0 2-3) (Tabela 1).

Tabela 1 - Mediana, $1^{\circ}$ e $3^{\circ}$ quartil das medidas da força muscular do assoalho pélvico pelo teste da AFA e perineômetro nos grupos pós-parto vaginal (G-PV) e pós-parto cesárea (G-PC) e nas nulíparas(G-NP).

\begin{tabular}{|c|c|c|c|}
\hline \multirow[t]{3}{*}{ Testes } & \multicolumn{3}{|c|}{ Grupos } \\
\hline & G-PV & G-PC & G-NP \\
\hline & Parto vaginal & Parto cesáreo & Nulípara \\
\hline AFA & $2,0[1,0 ; 2,0]^{*}$ & $2,0[2,0 ; 3,0]$ & $3,0[2,0 ; 3,0]^{*}$ \\
\hline Perineômetro $(\mathrm{mmHg})$ & $2,0[1,5 ; 2,5]^{*}$ & $2,0[2,0 ; 3,0]$ & $3,0[2,0 ; 3,0]^{*}$ \\
\hline
\end{tabular}

AFA = avaliação da força do assoalho pélvico

* = diferença significante em relação ao grupo G-NP

Tanto o parto vaginal como o parto cesárea aumentaram o RR de exame alterado da força muscular do AP, entretanto o IC a 95\% só foi significativo após o parto vaginal (Tabela 2).

Tabela 2 - Risco relativo (RR), IC a 95\% e valor de p de exame alterado da força da musculatura do assoalho pélvico avaliado pela AFA e perineômetro nos grupos pósparto vaginal (G-PV) e pós-cesárea (G-PC).

RR IC 95\% Valor

de $p$

AFA

G-PV - Pós-parto vaginal $2,58^{*} 1,32-5,04 \quad 0,002$

G-PC - Pós-parto cesárea 1,56 0,94-2,57 0,12

Perineômetro (mmHg) G-PV - Pós-parto vaginal 2,31* 1,24-4,32 0,005

G-PC - Pós-parto cesárea 1,38 $\quad 0,85-2,23 \quad 0,29$

AFA = avaliação da força do assoalho pélvico.

O número e o percentual de exames alterados e normais da força muscular do AP avaliados pela AFA e pelo perineômetro foram semelhantes, com coeficiente de kappa de 0,87 e IC 95\%: 0,780,96 (Tabela 3), evidenciando correlação entre ambos os exames.
Tabela 3 - Número e distribuição percentual de exames alterados e normais pela AFA e pelo perineômetro nas primíparas pós-parto vaginal (G-PV) e pós-parto cesárea (G$\mathrm{PC})$ e nas nulíparas (G-NP)

\begin{tabular}{|c|c|c|c|c|c|c|}
\hline \multirow[t]{5}{*}{ Variáveis } & & \multicolumn{5}{|c|}{ Grupos } \\
\hline & & \multicolumn{2}{|c|}{ G-PV } & \multicolumn{2}{|c|}{ G-PC } & G-NP \\
\hline & & \multicolumn{5}{|c|}{ Pós-parto Pós-parto Nulípara } \\
\hline & & \multicolumn{2}{|c|}{ vaginal } & \multicolumn{2}{|c|}{ cesárea } & \multirow[b]{2}{*}{ n $\%$} \\
\hline & & $\mathrm{n}$ & $\%$ & $\mathrm{n}$ & $\%$ & \\
\hline \multirow[t]{2}{*}{$\overline{A F A}$} & Alterados & 25 & 78,0 & 19 & 59,0 & 1127,0 \\
\hline & Normais & 7 & 22,0 & 13 & 41,0 & 1963,0 \\
\hline \multirow[t]{2}{*}{ Perineômetro (mmHg) } & Alterados & 24 & 75,0 & 17 & 53,0 & 1127,0 \\
\hline & Normais & 8 & 25,0 & 15 & 47,0 & 1963,0 \\
\hline
\end{tabular}
AFA = avaliação da força do assoalho pélvico.

\section{Discussão}

A força muscular do AP de primíparas diminuiu após 4-6 meses de parto vaginal. Houve também aumento do RR de exame alterado da força muscular, tanto na avaliação feita pela AFA quanto pelo perineômetro.

Os métodos de avaliação, AFA e perineômetro, da força muscular do AP foram escolhidos por serem os mais utilizados na prática clínica ${ }^{14}$. Os resultados evidenciam que foram métodos concordantes (kappa de 0,87 e IC a 95\% de 0,78 a 0,96), provavelmente influenciados pela forma padronizada de classificação adotada. Esses resultados evidenciam que o profissional com experiência pode usálos de modo aleatório, na avaliação da força muscular do AP. Entretanto, do ponto de vista de pesquisa, o uso do perineômetro deve ser indicado pela reprodutibilidade dos resultados e por sua objetividade.

A idade materna mais avançada é um fator que pode potencializar a lesão muscular e esta foi a razão da seleção de mulheres entre 20 e 30 anos. A distribuição semelhante dos grupos nas três faixas etárias (Figura 1) permite validar os resultados. É relatado que a função do músculo estriado diminui cerca de $1 \%$ ao ano, após pico na meia idade, e que esta diminuição cumulativa associase à lesão do tecido conjuntivo ${ }^{1}$.

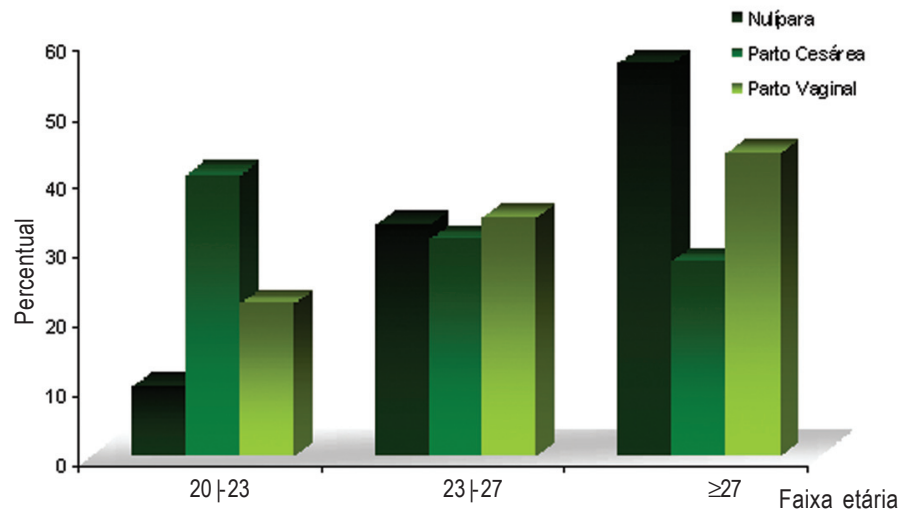

Figura 1 - Percentual de mulheres dos três grupos experimentais: nuliparas, pós-parto vaginal e pós-parto cesárea, nas faixas etárias de $20+23,23+27$ e $\geq 27$ anos $(p=0.06)$. 
$\mathrm{Na}$ gestação, a parede abdominal torna-se flácida devido ao estiramento e ao afastamento do músculo reto abdominal, evidenciado pela diástase desses músculos. A recuperação da tonicidade da musculatura da parede ocorre lenta e, às vezes, imperfeitamente ${ }^{20}$. Isto deve ocorrer também com a musculatura do AP, como evidenciado neste trabalho.

É interessante que nas pacientes que foram submetidas à cesárea, a força muscular do AP diminuiu para um valor intermediário entre a das nuliparas e as pós-parto vaginal e foi 1,5 vez maior o RR de exame alterado da força muscular do AP em mulheres submetidas à cesárea, porém o IC a 95\% não foi significativo. Esses resultados sugerem que não apenas o estiramento do AP causado pela passagem do feto mas também a embebição gravídica, a distensão da musculatura da parede abdominal causada pelo útero gravídico e o peso do feto distendendo a parede abdominal e o AP podem alterar a musculatura do AP.

Tanto a gestação quanto o parto são momentos de grandes mudanças anatômicas e fisiológicas para o organismo materno, em especial, para o trato urinário, podendo resultar em alteração na função, manifestada por incontinência urinária de esforço (IUE) ${ }^{21}$. Está bem documentado que o parto vaginal resulta em lesões nas estruturas anatômicas e nervosas do AP. Contudo, o parto cesáreo não protege as lesões das estruturas, em especial nas parturientes que atingiram o segundo período do parto (periodo expulsivo ou período pélvico), sugerindo que a gravidez e o trabalho de parto podem lesar o $\mathrm{AP}^{22}$.

Estudo prospectivo demonstrou que a cesárea é um dos fatores de risco mais importantes para o desenvolvimento do prolapso genital ${ }^{7}$. No estudo realizado com 363 mulheres, um ano depois do primeiro parto, a prevalência de IUE foi similar. Isto é possivel, porque as lesões no AP foram semelhantes nos dois $\operatorname{casos}^{23}$. Os resultados encontrados neste trabalho na força muscular diminuída do AP pós-cesárea confirmam essas afirmativas.

Os resultados encontrados neste trabalho mostram que o parto vaginal em primíparas diminuiu a força muscular do AP e aumentou o risco relativo de força muscular diminuída do AP, após 4 a 6 meses do parto, e que a cesárea não protegeu o AP. Allen et al. ${ }^{23}$ mostraram que mulheres que tiveram cesárea eletiva antes do início do trabalho de parto foram poupadas de lesão muscular do AP. Esta avaliação não foi realizada neste trabalho o que impediu a comparação com esses resultados $^{24}$.

A questão então não é apenas se o parto vaginal lesa o diafragma pélvico, mas também ava- liar o grau de lesão e se a episiotomia protege o AP. A freqüente alegação feita em apoio ao uso liberal da episiotomia é que ela previne a flacidez do AP durante o parto, prevenindo a IUE e o prolapso genital $^{25}$. A revisão sistemática da literatura ${ }^{25}$ evidencia que o uso liberal da episiotomia só foi vantajoso na diminuição do risco de trauma anterior do assoalho pélvico. Nossos resultados ratificam esses achados, pois mesmo com o uso rotineiro da episiotomia, a força muscular do AP diminuiu 4 a 6 meses pós-parto vaginal. O efeito da episiotomia médio-lateral na força do AP foi avaliado, após 3 meses de parto vaginal, pelo teste bidigital e perineometria vaginal, e os autores concluíram que a episiotomia foi responsável pela diminuição da força muscular do $\mathrm{AP}^{26}$. A episiotomia de rotina, e não apenas o parto vaginal, pode ter sido a causa dos nossos achados, pois todas as primiparas do Grupo G-PV foram submetidas à episiotomia.

Ficam dúvidas importantes a serem investigadas: se a cesárea realizada sem trabalho de parto prévio também diminui a força muscular do AP; se no final da gestação, há diminuição da força muscular do AP; se o uso da episiotomia de rotina influencia a força muscular do AP, após 4 a 6 meses do parto, e se alterações patológicas maternas, associadas com maior ganho de peso materno e macrossomia fetal, causam maior alteração da força muscular do AP. Essa dúvidas merecem investigação futura.

A análise e discussão dos resultados permitem concluir: o parto vaginal diminuiu a força muscular do AP de primíparas, avaliada pela AFA e pelo perineômetro, após 4 a 6 meses do parto; o parto vaginal em primiparas aumentou em 2,58 ou 2,31 vezes o RR de diminuição da força muscular do AP, após 4 a 6 meses do parto; o parto cesáreo em primíparas também aumentou em 1,56 ou 1,37 vezes o risco relativo de diminuição da força muscular do AP, após 4 a 6 meses do parto, porém o IC a $95 \%$ não foi significativo.

$\mathrm{Na}$ realidade, com os resultados obtidos, abre-se linha para novas investigações importantes para o nosso país, onde são elevadas as taxas de cesárea. É preciso definir, a longo prazo, se realmente essas taxas são abusivas ou se estão corretas e há benefício para as mulheres.

\section{Referências}

1. Polden M, Mantle J. Fisioterapia em ginecologia e obstetrícia. $2^{a}$ ed. São Paulo: Ed. Santos; 2000.

2. Meyer S, Schreyer A, De Grandi P, Hohlfeld P. The effects of birth on urinary continence mechanisms 
and other pelvic-floor characteristics. Obstet Gynecol. 1998;92(4 Pt 1):613-8.

3. Snooks SJ, Henry MM, Swash M. Faecal incontinence due to external anal sphincter division in childbirth is associated with damage to the innervation of the pelvic floor musculature: a double pathology. Br J Obstet Gynaecol. 1985;92(8):824-8.

4. Wijma J, Potters AE, de Wolf BT, Tinga DJ, Aarnoudse JG. Anatomical and functional changes in the lower urinary tract during pregnancy. BJOG. 2001;108(7):726-32.

5. Henry MM, Parks AG, Swash M. The pelvic floor musculature in the descending perineum syndrome. Br J Surg. 1982;69(8):470-2.

6. Smith AR, Hosker GL, Warrell DW. The role of pudendal nerve damage in the aetiology of genuine stress incontinence in women. Br J Obstet Gynaecol. 1989;96(1):29-32.

7. Groutz A, Rimon E, Peled S, Gold R, Pauzner D, Lessing JB, et al. Cesarean section: does it really prevent the development of postpartum stress urinary incontinence? A prospective study of 363 women one year after their first delivery. Neurourol Urodyn. 2004;23(1):2-6.

8. ACOG Committee on Ethics. ACOG committee opinion. Surgery and patient choice: the ethics of decisionmaking. Int J Gynaecol Obstet. 2004;84(2):188-93.

9. Moreira ECH. Valor da avaliação propedêutica objetiva e subjetiva no diagnóstico da incontinência urinária feminina: correlação com a força muscular do assoalho pélvico. Rev Bras Ginecol Obstet. 2000;22(9):597.

10.Kirschner-Hermanns R, Wein B, Niehaus S, Schaefer W, Jakse G. The contribution of magnetic resonance imaging of the pelvic floor to the understanding of urinary incontinence. $\mathrm{Br} \mathrm{J}$ Urol. 1993;72(5 Pt 2):715-8.

11. Contreras Ortiz O, Coya Nuñez F. Dynamic assessment of pelvic floor function in women using the intravaginal device test. Int Urogynecol J Pelvic Floor Dysfunct. 1996;7(6):317-20.

12. Hahn I, Milsom I, Ohlsson BL, Ekelund P, Uhlemann C, Fall M. Comparative assessment of pelvic floor function using vaginal cones, vaginal digital palpation and vaginal pressure measurements. Gynecol Obstet Invest. 1996;41(4):269-74.

13. Thorp JM, Jones LH, Wells E, Ananth CV. Assessment of pelvic floor function: a series of simple tests in nulliparous women. Int Urogynecol J Pelvic Floor Dysfunct. 1996;7(2):94-7.

14. Universidade de Málaga. Faculdade de Medicina. Bioestatística: métodos e aplicações. 2a ed. Málaga: Universidade de Málaga; 1997.

15. Briquet R, editor. Obstetrícia normal. 3a ed. São Paulo: Sarvier; 1994.

16. Oliveira Gameiro MOO, Amaro JL. Eletroestimulação intravaginal e exercícios perineais no tratamento da incontinência urinária feminina. Rev Urol Panam. 2002;14(2):56-9.

17. Amaro JL, Oliveira Gameiro MO, Padovani CR. Treatment of urinary stress incontinence by intravaginal electrical stimulation and pelvic floor physiotherapy. Int Urogynecol J Pelvic Floor Dysfunct. 2003;14(3):204-8.

18. Riegelman RK. Studying a study and testing a test: how to read the medical evidence. 4th ed. Philadelphia: Lippincott Williams \& Wilkins; 1999. p. 26-41.

19. Gillstrap III LC, Cunningham FG, Vandorsten JP. Operative obstetrics. 2nd ed. New York: McGrawHill; 2002. p. 63-88.

20. Chaliha C, Stanton SL. Urological problems in pregnancy. BJU Int. 2002;89(5):469-76.

21.Lal MH, Mann C, Callender R, Radley S. Does cesarean delivery prevent anal incontinence? Obstet Gynecol. 2003;101(2):305-12.

22. Benassi L, Bocchialini E, Bertelli M, Kaihura CT, Ricci L, Siliprandi V. Risk of genital prolapse and urinary incontinence due to pregnancy and delivery. A prospective study. Minerva Ginecol. 2002;54(4):31724.

23. Allen RE, Hosker GL, Smith AR, Warrell DW. Pelvic floor damage and childbirth: a neurophysiological study. Br J Obstet Gynaecol. 1990;97(9):770-9.

24. Schindl M, Birner P, Reingrabner M, Joura E, Husslein P, Langer M. Elective cesarean section vs. spontaneous delivery: a comparative study of birth experience. Acta Obstet Gynecol Scand. 2003;82(9):834-40.

25. Carroli G, Belizan J. Episiotomy for vaginal birth. Cochrane Database Syst Rev. 2004;(2):CD000081.

26. Sartore A, De Seta F, Maso G, Pregazzi R, Grimaldi E, Guaschino S. The effects of mediolateral episiotomy on pelvic floor function after vaginal delivery. Obstet Gynecol. 2004;103(4):669-73. 